\title{
DESIGN AND DEVELOPMENT OF LOAD SHARING MULTIPATH ROUTING PROTCOL FOR MOBILE AD HOC NETWORKS
}

\author{
K.V. Narayanaswamy ${ }^{1}$, C.H. Subbarao ${ }^{2}$ \\ ${ }^{1}$ Professor, Head Division of TLL, MSRUAS, Bangalore, INDIA, ${ }^{2}$ Associate Professor, CSE Dept, QCET Nellore, AP \\ ${ }^{1}$ drkvn21@gmail.com, ${ }^{2}$ chepurusubbarao@gmail.com
}

\begin{abstract}
Routing in Mobile Ad hoc Networks (MANETs) is a challenging task due to the dynamic nature of the network topology and resource constraints. Due to communication over wireless channel, participating nodes also experience interference and bandwidth constraints. Therefore it is essential to develop a robust and efficient routing protocol for MANETs. This research paper involves design and development of a multipath routing protocol for MANETs, called Load Sharing Multipath Routing (LS-MPR) uses dual polarized directional antenna to enhance network efficiency and provide load balancing. LS-MPR is an on-demand multipath routing protocol, which selects best possible multiple paths based on ascending order of hop count and availability of common polarization between neighboring mobile nodes. The performance of the LS-MPR is compared with that Ad-hoc On Demand Distance Vector (AODV) and Dynamic Source Routing (DSR).

The network performance is checked with and without node mobility, throughput, Packet Delivery Ratio (PDR), least jitter, low interference and low end-to-end delay. Directional antenna is a very efficient and low cost smart antenna technology. It can achieve better performance, higher throughput, and better resource utilization with omnidirectional antennas multi-path routing cannot be exploited very well since packets routed on one of the paths cause an interference zone that typically encompasses the other paths and thereby limits the number of packets routed on these paths.
\end{abstract}

Keywords: Multipath Routing, Load Sharing Multipath Routing (LS-MPR) Ad-hoc On Demand Distance Vector (AODV) Dynamic Source Routing (DSR).

\section{INTRODUCTION}

The multipath routing could offer several benefits like load balancing, fault-tolerance, higher aggregate bandwidth, lower end-to-end delay, security, energy-conservation, and QoS. Load balancing is of special importance in MANETs because of the limited bandwidth between the nodes general scenario of mobile Ad-hoc network is shown in Fig-1. The key techniques that can be used to overcome these problems are smart antennas and modified MAC layer and routing protocols. With directional antennas it is now possible to construct disjoint paths that do not interfere with each other and having dual polarized directional antenna can improve the efficiency of mobile node.

Pro-active protocols or table-driven protocols works in a way similar as wired networks. They try to maintain an up-to-date map of the network, by continuously evaluating known routes and attempting to discover new ones. There is no extra delay due to route discovery as route is already known. But keeping the information up-to-date may require a lot of bandwidth, which is sparse, and battery power.

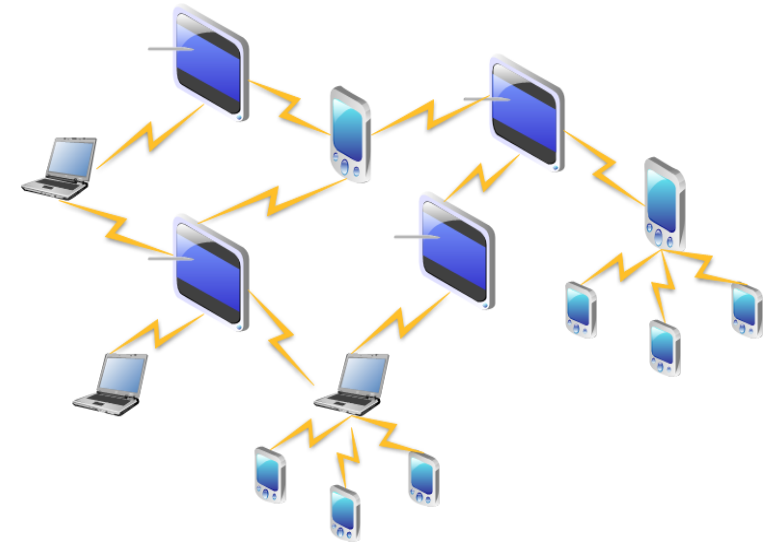

Fig-1: Mobile Ad-hoc Network Scenario

Pro-active protocol only starts a route discovery procedure when needed. This does not require the constant updates being sent through the network, as in pro-active protocols, but it does cause delays, since the routes are not available and need to be found. In some cases the desired route(s) are still in the route cache maintained by nodes. When this is the case there is no additional delay since routes do not have to be 
discovered. Therefore, on-demand protocols are more scalable to dynamic, large networks. On demand protocols consist of the following three main phases.

1. Route discovery is the process of finding a route between source node and destination node.

2. Route Recovery is the process of finding routes whenever route link breaks, or node moves out from network. 3. Route maintenance is the process of repairing a broken route or finding a new route in the presence of a route failure.

Related work for multipath routing using directional antenna and omnidirectional antenna has been done by many researchers to study the effect of directional antenna on multipath routing. Reference [1] Presents Split Multipath Routing (SMR) protocol that builds maximally disjoints paths. Multiple routes, of which one is the shortest delay path, are discovered on demand.

[2] Suggested of using directional antennas to improve the efficiency of on-demand routing protocols in mobile ad hoc networks. The idea is to use directional re-broadcasting during the route re-discovery process.

[3]The directional antennas were used to improve routing performance in two situations. One was in the case of dynamic network partitioning due to mobility, and the other was during route repair process caused by the movement of intermediate node.

[4] A simple MAC protocol named DiMAC was proposed and the DSR routing protocol was evaluated based on DiMAC it is based on IEEE 802.11 DCF and it also uses RTS and CTS for channel reservation.

[5] DiMAC is proposed and the DSR routing protocol is evaluated based on DiMAC. Several modifications are also introduced to improve DSR performance for directional transmissions. Because of the unsolved "deafness" problem,

[6] They have presented the initial design and evaluation of two techniques for routing improvement using directional antennas in mobile ad hoc networks. Their design and evaluation is based on the DSR protocol, an on-demand routing protocol for mobile ad hoc networks.

[7] Described the three different multipath routing protocols such as ZRP, AODV-BR and TORA. Multipath routing allows intermediate nodes to responds to RREQs, which can reduce the time for route discoveries.

No researchers has described about dual polarised directional antenna and its effect on multipath routing. This paper explains the effect of dual polarised directional antenna for multipath routing MANETs and its use for increasing the throughput.

\section{DESCRIPTION}

A multipath routing protocol which is reactive in nature is described in this paper by using dual polarised directional for MANETs. The importance of this protocol is to provide load balancing, by sharing load on to multiple paths.

\section{DESIGN REQUIRMENTS}

To achieve the required performance different requirements need to be taken into the design specifications which are shown below.

FNR_1: Low Interference: There should be low interference to achieve high throughput. This FNR_1 can be accomplishing by using design specifications of Directional Antenna with Dual Polarisation [Dependency None].

FNR_2: Low Routing Overhead: The protocol must be a reactive routing protocol to accomplish low routing overhead in the network [Dependency FNR_3].

FNR_3: Reliable Routing: The protocol must provide reliable routing in terms of route breakages, link breakage or change in the topology. Concurrent parallel multipath, sufficient nodal residual power, etc. are design specification to complete FNR_3 [Dependency FNR_2].

FNR_4: Low End to End Delay: The protocol ought to low end to end delay by minimizing number of hops [Dependency None].

FNR_5: Low Jitter: The protocol should have low jitter to get rid of noise in the network; this can be attained by reducing number of hops [Dependency None].

FNR_6: Efficient Multipath Routing: The protocol must make available of multipath routing, can be done by optimizing the number of hops.

NFR1: The protocol must be scalable in terms of network size and hop count

NFR2: The protocol must be able to work even if the nodes are mobile.

\subsection{APPROACH \& methodology}

Design of Multipath Routing: The design specifications for the multipath routing algorithm using dual polarized directional antenna is arrived at based on the reviewed literature and performance requirements for selected routes.

Development of Multipath Routing: The designed multipath routing protocol is implemented in Qualnet such that multiple routes are chosen from a source to destination based on polarization.

Testing and Validation of Multipath Routing: A suitable test scenario is developed to test the performance of the implemented protocol. The performance of the protocol is compared with the obtained results to check, throughput, latency and end to end delay. 


\section{SOLUTION PROCEDURE}

While AODV protocol was selected to provide multipath routing. LS-MPR was new proposed multipath routing protocol which was simulated through command line. To design and develop LS-MPR V model was used as shown in Fig-2.

The design of multipath routing is divided into high- level design and low level design. High level design as shown in Fig-3. There are two parts of this block, neighbor discovery and route establishment and data exchange. When a node want to start communication to any other node in the network first it has to know about its neighbour, this process is called as neighbour discovery. This can be done by exchange of broadcast message. Broadcast packet information consists of ten indices.

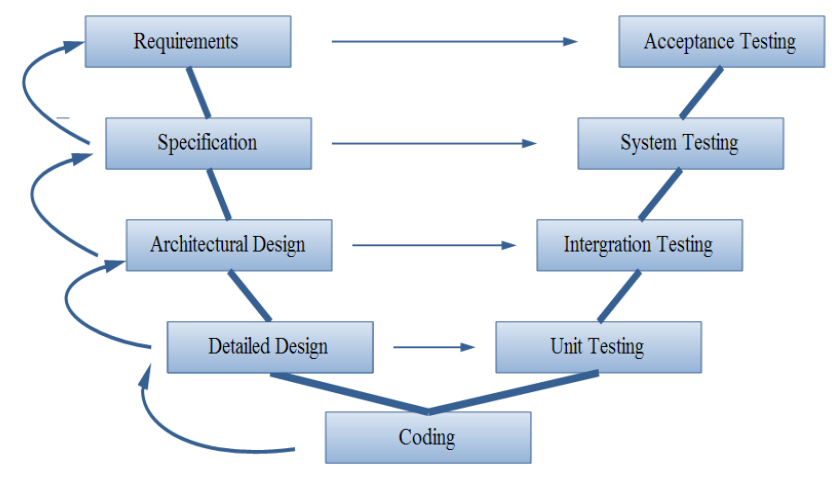

Fig-2: Verification and Validation Model

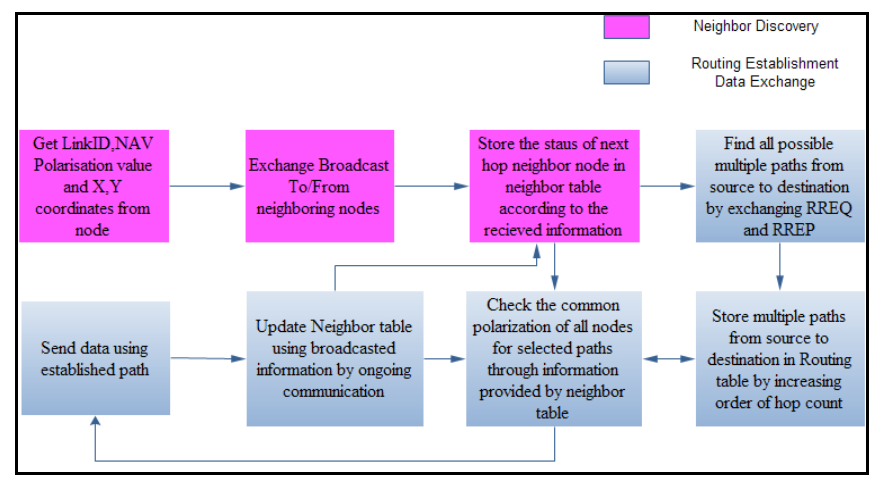

Fig-3 Block Diagram of Multipath Routing

Each of the two parts consists of five indices. First five indices states the self- node, next is neighbour node or participated node, its status about polarisation, NAV value and $\mathrm{x}, \mathrm{y}$ coordinates.

Example of idle node in network: $(1,1, \mathrm{vF}, \mathrm{NAV},(200,300)$; 1, 1, hF, NAV, (200,300))
Example of busy node in network: $(2,1, \mathrm{vB}, \mathrm{NAV},(200,400)$; 2, 2, hF, NAV, $(200,400))$

Neighbor discovery consist of three blocks. Whenever any mobile node comes into the network, its neighbour should know about its link id, Polarisation status, NAV value and $\mathrm{x}, \mathrm{y}$ coordinate. This information is helpful to prepare the neighbour table.

Neighbor table will store the information about next hop node according to the received broadcast message. After this, source will initiate the route request, RREQ will received by the neighbor node with the broadcast message information and RREP will be generated to find all possible multiple path from source to destination.

\subsection{Low Level Design of Multipath Routing Protocol:}

Fig-3 shows flowchart for route establishment and data exchange; Source will initiate route request to intended destination, first it will check into the routing table for availability of path. If path is available, find best possible path according to the criteria specified above and send the data. If path is not available or best path is not there then broadcast route request with header information, wait for route reply till threshold time. If RREP not received within threshold start new route request. This will update neighbour table after selection of best path.

Fig-4 and Fig-5 demonstrates flowchart for selecting best path for data exchange, When source node receives RREP from all the neighboring nodes, it will save all the routes into the routing table, in descending order of hop count, so that least hop count will have highest priority. If common polarisation found between all nodes till destination then sends the data Otherwise check the NAV period to below threshold, if it is below threshold then wait till NAV gets over otherwise send error message route not found. Source will check in the database for alternate route to the destination.

\subsection{Implementation of Multipath Routing Protocol:}

The implementation of the protocol is done by considering the steps of the design that have been carried out in discrete event simulator used to simulate the developed routing protocol by doing code level changes in physical layer, MAC layer and network layer using $\mathrm{C}$ and $\mathrm{C}++$.

\subsection{Testing of Developed Multipath Routing Protocol:}

Testing and validation of the implemented protocol is carried out for different scenarios like the functional requirements, Unit testing, integration testing, System testing and Acceptance testing are conducted. 


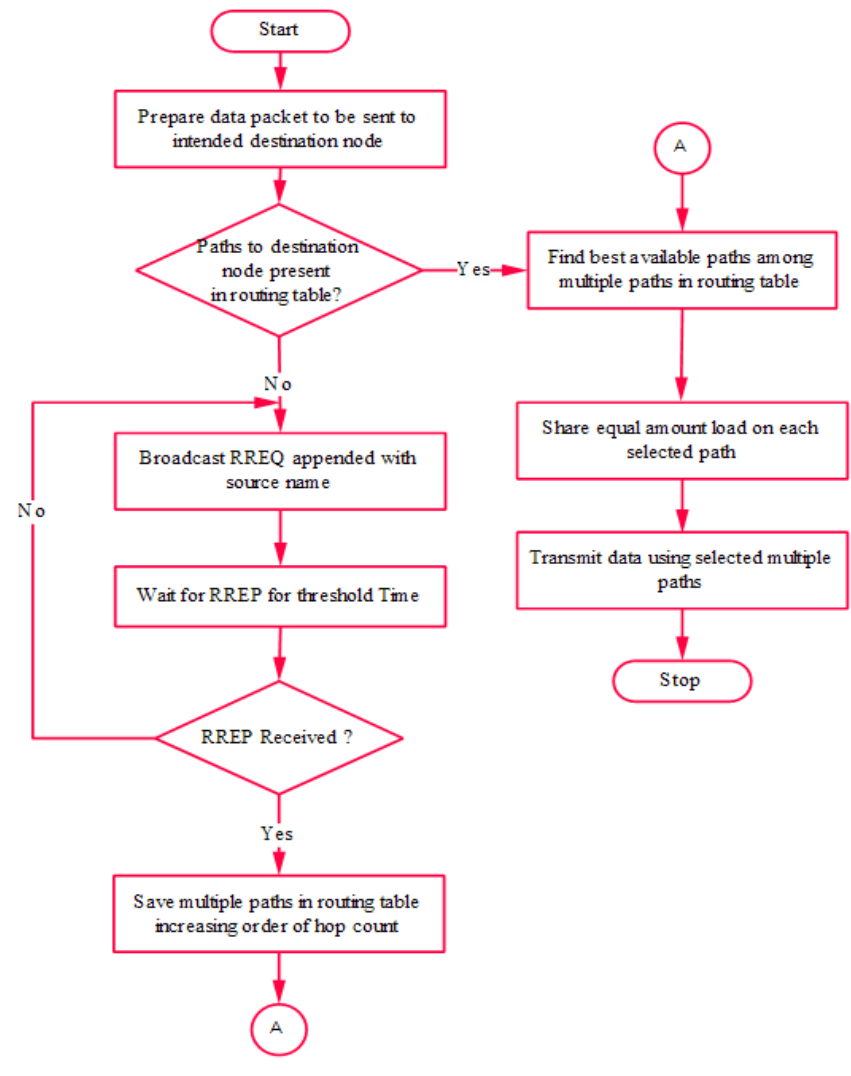

Fig-4: Source Searching For Paths to Destination

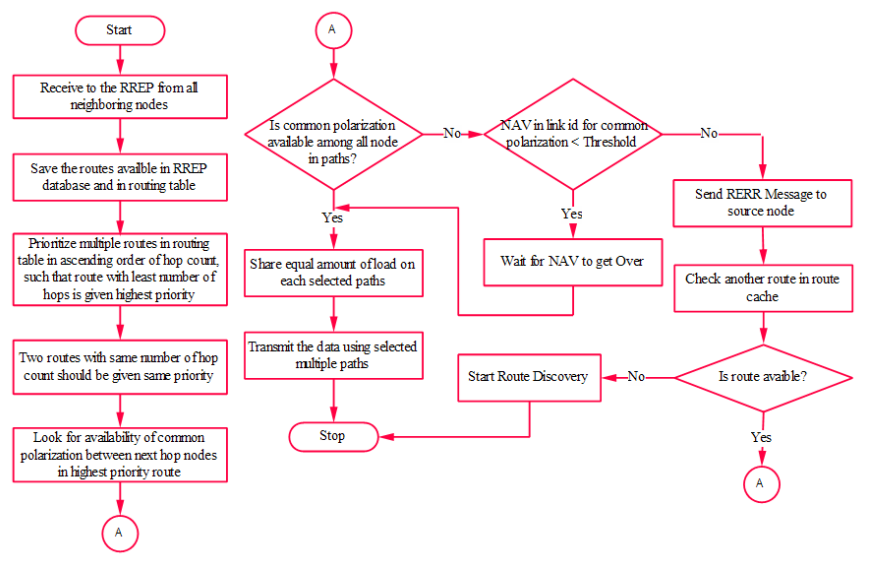

Fig-5: Selection of path for data exchange

\section{RESULTS AND DISSCUSSIONS}

The performance of the LS-Multipath Routing Protocol is tested based on different performance metrics like throughput, latency, Packet Delivery Ratio (PDR), end to end delay and jitter.
Fig-6 shows the scenario for communication between nodes using Dual Polarisation with Directional antenna (DP-DA), azimuth and elevation antenna pattern. In this scenario node 1 and node 4 have both antennas pattern i.e. DP-DA. and illustrates the four step communication of DP-DA for node 1 and node 4 .

Also Fig-6 shows node 1 has both antenna pattern (azimuth and elevation) and node 4 has only (elevation pattern). So when they want to start communication node 1 send RTS in elevation pattern (D), as node 4 has only elevation antenna pattern, CTS send in only elevation (e), Data is sent in elevation antenna pattern (D) and Acknowledgement also goes in elevation antenna pattern.

\subsection{Implications of Unit Integration \&System Testing:}

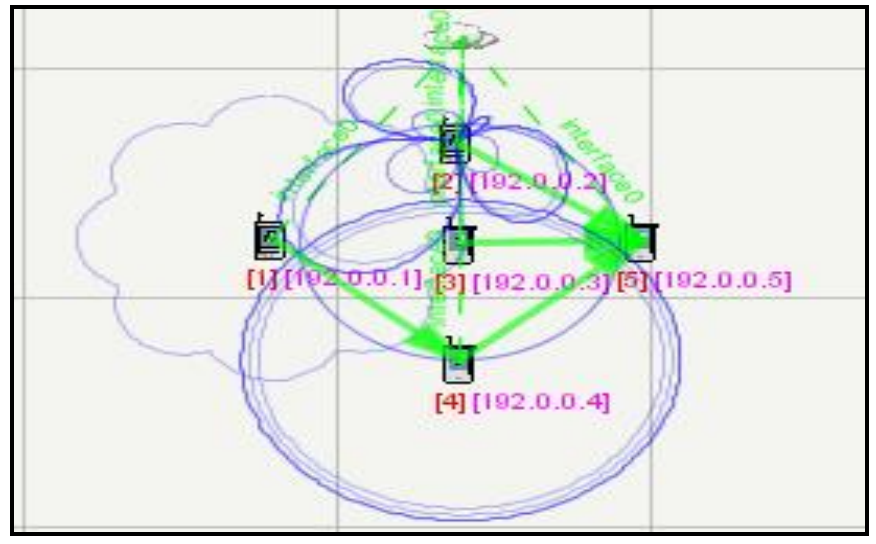

Fig-6: Scenario for DP-DA communication

\subsection{Performance Testing:}

Performance testing has carried out for static multipath routing. In static multipath routing maximum number of nodes is tested is from 2 to 15 . The result is shown only for number of nodes 10 . Terrain size is defined to 1500 X $1500 \mathrm{~m}$, Traffic type is selected to Constant bit Rate (CBR). Mobility model is selected random way point and none, to test the performance with mobility and no mobility.

Total packet send is 72 over three nodes, each node carrying 36 packets and destination will receive total 72 packets, as two paths has been defined statically. Each packet size is considered to 512 bytes with simulation time of 600 seconds. The distance between nodes has been kept static to $260 \mathrm{~m}$ when there is no mobility. Two reactive routing protocols have been considered for performance testing AODV and DSR with LS-MPR. Path design criteria to have node disjoint paths. The results are tabulated and are shown in Table-1. 
Table-1: Result of Dual Polarised antennas in communication for Scenario of Fig-6

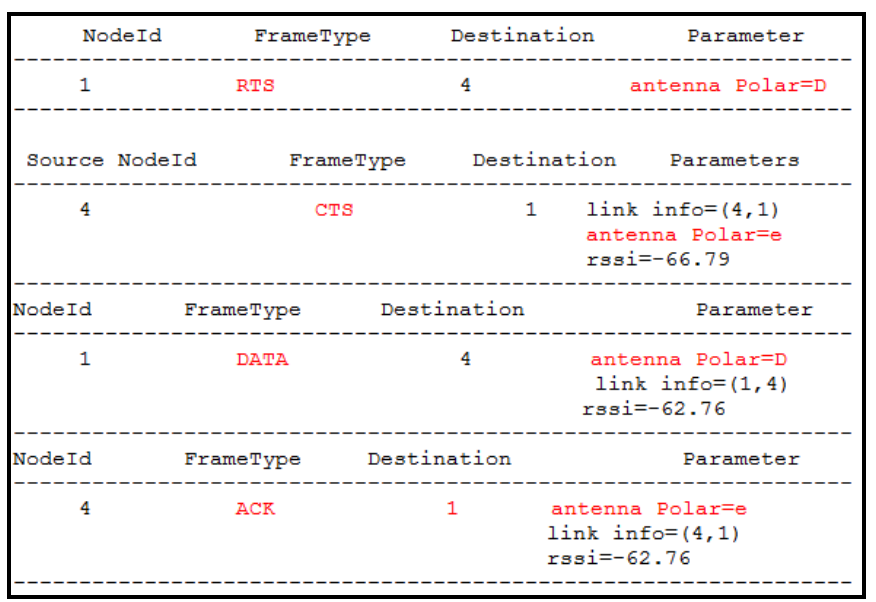

Fig-7 Illustrates the static multipath routing for 10 nodes, with source node 1 and destination node 10 . Two paths have been selected by method of finding multiple paths. Two paths are as follows $[(1,2,4,6,8,10),(1,3,5,7,9,10)]$, simultaneous packet transmission can be seen from node 2 to node 4 , from node 3 to node 5 , if any node cannot send to further node, it will buffer and send.

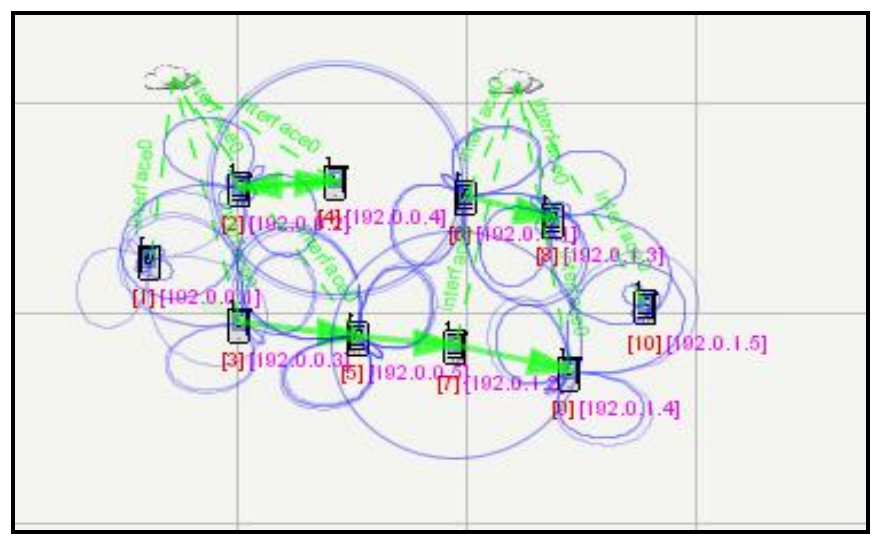

Fig-7: Scenario for LS - Multipath routing

Chart-1: Describes the result for three reactive routing protocols, AODV, DSR and LS-MPR for total number of nodes 10. Comparison has been done with jitter, throughput and latency when there is no mobility. Jitter and Latency calculation is shown with E-2 and throughput calculation in E+3. Throughput with directional antenna for AODV and DSR is almost same to $4.23 \mathrm{E}+3$ and for LS-MPR it is around $4.5 \mathrm{E}+3$ which are high compared to both. Jitter was found to be almost same in all three protocols to $\sim 0.5 \mathrm{E}-2$.
Latency is more in LS-MPR as it is static multipath routing, so the packet is being send simultaneously. Throughput calculation has been done iteratively at each node.

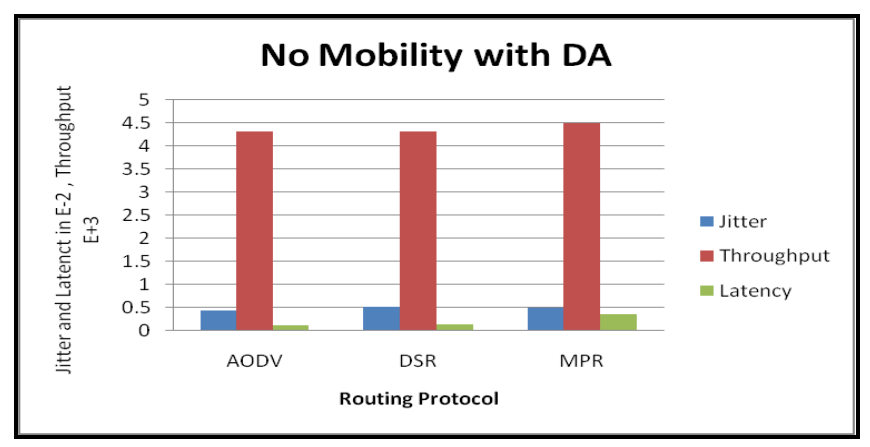

Chart-1: Results for directional antenna and with no mobility

Chart-2: Represents the results of three routing protocol when there was mobility with directional antenna. With mobility it has found that latency has been increased highly in all three routing protocols. DSR has less latency compared to other two protocols. Jitter is equal in DSR and LS-MPR to 0.4E-2, whereas in AODV it was 1.1E-2. Throughput is achieved high in LS-MPR compared to other both, the reason being that as throughput has calculated iteratively and CBR link was established between each node.

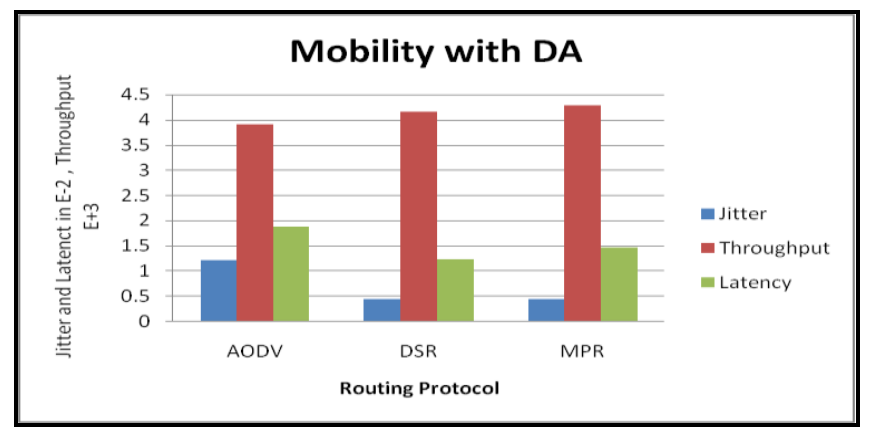

Chart-2: Results for Mobility and with directional antenna

Chart-3: Illustrates the effect of dual polarized directional antenna on multipath routing with mobility and without mobility. As dual polarized directional antenna can deal with the problem of interference or Jitter and this can be seen in Figure 10. End to End delay also found less in all the routing protocols described in this graph. Packet delivery ratio also found high compared to directional antenna. For AODV throughput was achieved around $4.45 \mathrm{E}+3$ and DSR and LSMPR were achieved more than $4.5 \mathrm{E}+3$. 


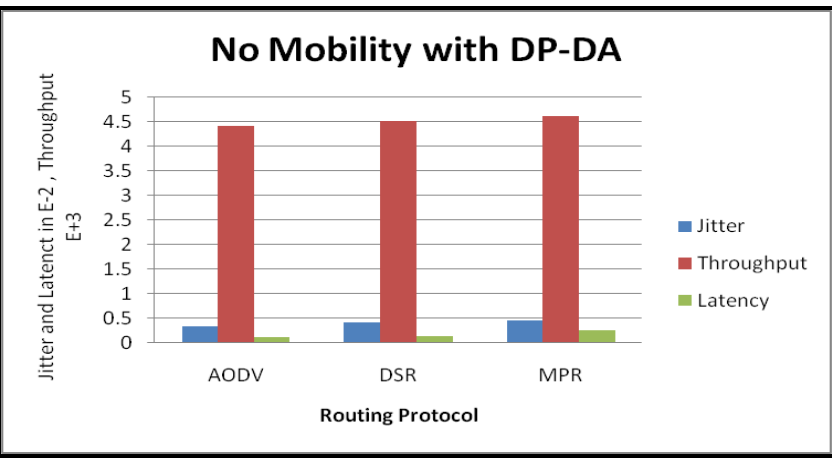

Chart-3: Results for no mobility and DP-DA

Chart-4: Represents the graph result of DP-DA with mobility for 10 nodes have multiple path as LS-MPR and AODV and DSR as unipath routing. It can be seen that when there was mobility with AODV protocol, throughput was achieved $4.1 \mathrm{E}+3$ but DSR and LS-MPR has got throughput around $4.4 \mathrm{E}+3$ and $4.5 \mathrm{E}+3$. It can be concluded though there is mobility, LS-MPR achieves higher throughput compared to other protocols. Latency was found high in AODV compared to other protocol. Jitter was found less in LS-MPR reason being that it was using multipath routing, so packets were send simultaneously.

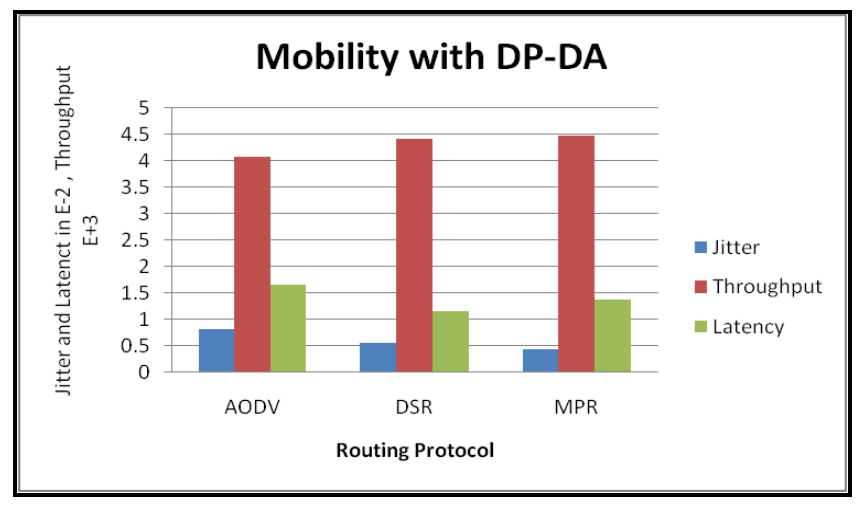

Chart-4: Results for mobility and with DP-DA

\section{CONCLUSIONS}

In this paper, a multipath routing protocol for MANETs using dual polarised directional antenna with load sharing, called LS-Multipath Routing protocol is designed and developed. This design requirement was achieved by using the dual polarised directional antenna to accomplish low interference, reduced end to end delay and multipath algorithm used to get multiple paths. Link breakage is avoided by updating routing table as well as neighbour table. It is found that the latency is considerably reduced when compared to other reactive protocols like DSR and AODV.

\section{REFERENCES}

[1] Lee S. J., Gerla M., Split Multipath Routing with Maximally Disjoint Paths in Ad Hoc Networks, In Proceedings of the IEEE International Conference on Communications, pp.3201-3205, 2001.

[2] Nasipuri Asis., Mandava J., Manchala H., Hiromoto R.E., On Demand Routing Using Directional Antennas in Mobile Ad Hoc Networks, In Proceedings of the IEEE WCNC 2000, pp.535 - 541, 2000.

[3] Saha A. K., Johnson D. B., Routing Improvement using Directional Antennas in Mobile Ad-hoc Networks, Global Telecommunications Conference, GLOBECOM '04. Vol.5, pp.2902 - 2908, 2004.

[4] Choudhury R., Vaidya N., Performance of Ad-hoc Routing using Directional Antennas'. In Proceedings of the $2^{\text {nd }} \mathrm{ACM}$ international symposium on Mobile Adhoc Networking \& Computing, Newyork, USA, pp.95105, 2001.

[5] Choudhury R., Vaidya N. Impact of Directional Antennas on Ad Hoc Routing, 8th Conference on Personal and Wireless Communication (PWC) 2003, Venice, September 2003

[6] Saha A. K., Johnson D. B, Energy Saving and Parition Bridging using Directional Antennas in Mobile Ad hoc Networks, Rice University, 2003.

[7] Yang Li., Hong Man, Jin Yu, Yu Dong Y., Multipath Routing in Ad Hoc Networks Using Directional Antennas, IEEE/Sarnoff Symposium on Advances in Wired and Wireless Communication, pp.119-122, 2004.

[8] Johnson D. The Dynamic Source Routing Protocol for Mobile Ad Hoc Networks for IPv4 http://www.ietf.org/rfc/rfc4728.txt, Retrieved on $12^{\text {th }}$ Dec 2011.

[9] Perkins C., Das S. Ad hoc On-Demand Distance Vector Routing, http://www.ietf.org/rfc/rfc3561.txt, retrieved on 20 Oct. 2011. 